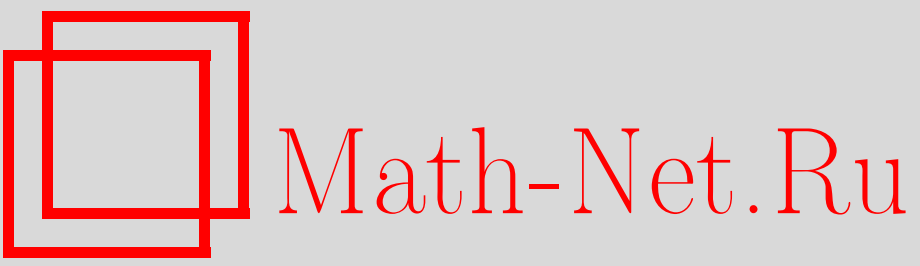

А. В. Булинский, Алгебраические $K$-системы и полупотоки сдвигов Пауэрса, УМH, 1996, том 51, выпуск 2, 145-146

DOI: https://doi.org/10.4213/rm948

Использование Общероссийского математического портала Math-Net.Ru подразумевает, что вы прочитали и согласны с пользовательским соглашением

http://www.mathnet.ru/rus/agreement

Параметры загрузки:

IP: 54.166 .219 .16

26 апреля 2023 г., 10:40:41 


\title{
АЛГЕБРАИЧЕСКИЕ $K$-СИСТЕМЫ И ПОЛУПОТОКИ СДВИГОВ ПАУЭРСА
}

\author{
А. В. Булинский
}

Некоммутативные (или квантовые) $K$-системы были введены в 70-е гг. Ж. Эмхом [1] как обобщение классических динамических систем (ДС), изучавшихся А. Н. Колмогоровым и Я. Г. Синаем. Тогда же и позднее были предложены различные концепции некоммутативной динамической энтропии, см., например, [2]. Теперь различают понятие алгебраической $K$-системы [3], восходящее к [1], и энтропийной $K$-системы, совпадение которых установлено при дополнительных свойствах динамики. Имея в виду проблему исследования инвариантов ДС, ограничимся здесь рассмотрением простых алгебраических характеристик. В данной работе, продолжающей [4], указано соответствие между $K$-системами Эмха и введенными Р. Пауэрсом в середине 80-х гг. полупотоками сдвигов операторных алгебр, см. [5].

Нам понадобится ряд определений. Пусть ДС $\left(M,\left(\beta_{t}\right)_{t \in T}\right)$ образована $W^{*}$-алгеброй $M$ с единицей 1 и (полу)группой вполне положительных нормальных отображений $\beta_{t} \in \mathscr{L}(M)$; параметр (время) $t \in \mathbb{R}$ (или $\mathbb{R}_{+}$) и $\beta_{s} \beta_{t}=\beta_{s+t}$ при $s, t \in T ; \beta_{0}=\operatorname{Id}_{M}$. При $T=\mathbb{R}$ все $\beta_{t} \in \operatorname{Aut}(M)$ и ДС обратима во времени. Динамика ( $\sigma$-слабо) непрерывна, если непрерывны все функции $t \mapsto \varphi\left(\beta_{t} a\right), \varphi \in M_{*}, a \in M$. Непрерывную полугруппу $\left(\beta_{t}\right)_{t \geqslant 0} \subset \operatorname{End}(M)$, следуя [5], именуем $E_{0}$-полугруппой, а $\beta \in \operatorname{End}(M)$ назовем сдвигом Пауэрса, если $\bigcap_{n \in \mathbb{N}} \beta^{n} \mu=\mathbb{C 1}$. Непрерывным полупотоком сдвигов назовем $E_{0}$-полугруппу, которая при $t>0$ состоит из сдвигов (термин Пауэрса - "поток сдвигов"), и рассмотрим далее дилатации (продолжения) такого полупотока до обратимого потока на объемлющей алгебре. Назовем $\beta \in \mathscr{L}(M)$ совместимым с точным нормальным состоянием (т.н.с.) $\omega \in M_{*}$, если (I) $\omega \circ \beta=\omega$ (инвариантность $\omega$ ), и вполне совместимым, если также (II) $\sigma_{t}^{\omega} \beta=\beta \sigma_{t}^{\omega}, t \in \mathbb{R}$, где $\left(\sigma_{t}^{\omega}\right)$ - группа модулярных автоморфизмов (ГМА), отвечающих $\omega$.

Алгебраическая $K$-система Эмха $\left(\mathfrak{M},\left(\alpha_{t}\right)_{t \in \mathbb{R}}, \varphi ; \mathfrak{N}\right)$ характеризуется наличием $W^{*}$-подалгебры $\mathfrak{N}\left(K\right.$-подалгебры), такой, что 1) $\left.\left.\alpha_{t} \mathfrak{N} \supset \mathfrak{N}, t>0,2\right) \bigvee_{t \geqslant 0} \alpha_{t} \mathfrak{N}=\mathfrak{M}, 3\right) \bigcap_{t<0} \alpha_{t} \mathfrak{N}=\mathbb{C} 1$, 4) для ГМА $\sigma_{t}^{\varphi} \mathfrak{N} \subset \mathfrak{N}, t \in \mathbb{R}$. Эквивалентность ДС $\left(M^{(k)},\left(\beta_{t}^{(k)}\right)_{t \in T}, \omega^{(k)}\right), k=1,2$, подразумевает сопряженность посредством $W^{*}$-изоморфизма $\gamma: M^{(1)} \rightarrow M^{(2)}$, сплетающего динамики $\left(\gamma \beta_{t}^{(1)}=\beta_{t}^{(2)} \gamma, t \in T\right)$ и сохраняющего т.н.с. $\left(\omega^{(2)} \circ \gamma=\omega^{(1)}\right) ;$ последнее условие излишне для полупотоков сдвигов. От эквивалентных $K$-систем из-за неединственности $K$-подалгебр требуем еще, чтобы $\gamma^{(1)}=\mathfrak{N}^{(2)}$ для выделенных подалгебр. ДС $\left(\mathfrak{M},\left(\alpha_{t}\right)_{t \in \mathbb{R}}, \varphi\right)$ является дилатацией $\left(M,\left(\beta_{t}\right)_{t \geqslant 0}, \omega\right)$, если сушествуют нормальные вложения $j: M \rightarrow \mathfrak{M}$ и проекция $p: \mathfrak{M} \rightarrow M$ такие, что $\varphi \circ j=\omega, \omega \circ p=\varphi, p \circ j=\operatorname{Id}_{M}$ и $\beta_{t}=p \alpha_{t} j, t \geqslant 0$. Дилатация минимальна, если $\alpha_{t} j M$ порождают $\mathfrak{M}\left(\mathfrak{M}=\bigvee_{t} \alpha_{t} j M\right)$. Марковость дилатации понимаем в смысле [6].

Примеры [5] полупотоков сдвигов на факторах типа $\mathrm{I}_{\infty}$ и $\mathrm{II}_{1}$ получены поднятием полупотоков сдвигов в гильбертовом пространстве на $C^{*}$-алгебру антикоммутационных соотношений и отвечают представлениям алгебры по (неточному) фоковскому состоянию и следу (с тривиальной ГМА). Обобщение конструкции (сравн. с [7]) дает нам модельные примеры связи сдвиговых систем.

ТеОрема 1. Непрерывный квазисвободный полупоток сдвигов Пауәрса, вполне совместимый с т.н.с., существует на каждом гиперконечном факторе типа $\mathrm{III}_{\lambda}$, $0<\lambda<1$. Такая ДС имеет минимальную марковскую дилатацию до квазисвободной К-системь Эмха, которая остается $К$-системой при обращении времени.

Заметим, что конструкция теоремы 1 дает два однопараметрических семейства ДС $\left(M^{(\nu)},\left(\beta_{t}^{(\nu)}\right)_{t \geqslant 0}, \omega^{(\nu)}\right)$, где $\nu \in(0,1 / 2)$ или $\nu \in(1 / 2,1)$. При этом $\lambda=\nu(1-\nu)^{-1}$, $\nu \in(0,1 / 2)$ и $\lambda=\nu^{-1}(1-\nu), \nu \in(1 / 2,1)$. Такие полупотоки сдвигов эквивалентны при условии $\nu_{2}=1-\nu_{1}, 0<\nu_{1}<1 / 2$, т.е. когда действуют на изоморфных факторах типа III . .

Разумеется, для обратной динамики $K$-подалгебра будет другой. В общем случае неквазисвободных ДС инвариантность $K$-свойства при обращении времени не имеет места, однако справедлива

Работа выполнена при поддержке Международного научного фонда и Правительства России (грант № J6M100). 
Лемма 1. $K$-система $\left(\mathfrak{M},\left(\alpha_{t}\right)_{t \in \mathbb{R}}, \varphi ; \mathfrak{N}\right)$ порождает непрерывный полупоток сдвигов $\beta_{t}=\left.\alpha_{-t}\right|_{\mathfrak{N}}, t \geqslant 0$, вполне совместимый $с \omega=\left.\varphi\right|_{\mathfrak{N}}$.

$K$-подалгебра $\mathfrak{N}$ инвариантна относительно $\alpha_{-t}, t \geqslant 0$, и неинвариантна относительно $\alpha_{t}$, $t>0$. В [3] рассмотрена "срезка" на $\mathfrak{N}$ динамики $K$-системы $\left(\alpha_{t}\right)_{t \geqslant 0}$, порождающая полугруппу $\left(\tau_{t}\right)_{t \geqslant 0} \subset \mathscr{L}(\mathfrak{N})$. Рассмотрим связь $\left(\tau_{t}\right)$ со сдвигами $\left(\beta_{t}\right)$. ДС $\left(M,\left(\tau_{t}^{+}\right)_{t \in T}, \omega\right)$ назовем ко-системой для $\left(M,\left(\tau_{t}\right)_{t \in T}, \omega\right)$, если (полу)группы $\left(\tau_{t}^{+}\right)$и $\left(\tau_{t}\right)$ сопряжены относительно $\omega$, т.е. $\omega\left(\tau_{t}(a) b\right)=\omega\left(a \tau_{t}^{+}(b)\right)$ при всех $a, b \in M, t \in T[6]$. Полная совместимость $\left(\tau_{t}\right)_{t \in T}$ с т.н.с. $\omega$ является критерием существования ко-(полу)группы $\left(\tau_{t}^{+}\right)_{t \in T}$.

ЛЕмма 2. Для обратимой динамики $\alpha_{t}^{+}=\alpha_{-t} \in \operatorname{Aut}(M)$ nри всех $t \in \mathbb{R}$, а для необратимой $\tau_{t}^{+}=\tau_{t}^{\prime \prime}, t \geqslant 0$, m.е. ко-полугруппа совпадает с бидуальной полугруппой $\left(\tau_{t}^{\prime \prime}\right)_{t \geqslant 0}$, введенной в [8] при условии совместимости $\left(\tau_{t}\right)_{t \geqslant 0}$ c m.н.с. $\omega$, задающим стандартное представление $M$.

Теорема $3[4]$ и следствие 3.2 .9 [6] показьвают, что для существования у ДС $\left(M,\left(\tau_{t}\right)_{t \geqslant 0}, \omega\right)$ минимальной дилатации до $K$-системы Эмха необходимо и достаточно, чтобы ее ко-система была полупотоком сдвигов Пауэрса.

Лемма 3. Дилатация Арвесона-Кишимото [9], примененная $к$ $E_{0}$-полугруппе $\left(\beta_{t}\right)_{t \geqslant 0} \subset \operatorname{End}(M)$, вполне совместимой с т.н.с. $\omega \in M_{*}$, определяет минимальную марковскую дилатацию $\left(M,\left(\beta_{t}\right), \omega\right)$ до обратимой ДС $\left(\mathfrak{M},\left(\alpha_{t}\right)_{t \in \mathbb{R}}, \varphi\right)$. При этом ко-ДС $\left(\mathfrak{M},\left(\alpha_{-t}\right)_{t \in \mathbb{R}}, \varphi\right)$ есть минимальная марковская дилатация для $\left(M,\left(\beta_{t}^{+}\right)_{t \geqslant 0}, \omega\right)$.

Пусть $j$ - вложение $M$ в $\mathfrak{M}$, определяющее продолжение, упомянутое в лемме 3 .

Лемма 4. В условиях леммы 3 , если $\left(\beta_{t}\right)_{t \geqslant 0}$ есть полупоток сдвигов Пауәрса, то $\left(\mathfrak{M},\left(\alpha_{-t}\right)_{t \geqslant 0}, \varphi ; \mathfrak{N}\right)$ есть K-система Эмха с K-подалгеброй $\mathfrak{N}=j(M)$.

Отсюда выводим утверждение, анонсированное в [10]: между классом $K$-систем и классом полупотоков сдвигов, вполне совместимых с т.н.с. $W^{*}$-алгебры, существует каноническое соответствие.

Теорема 2. Эквивалентные $K$-системы Эмха определяют әквивалентные непрерывные полупотоки сдвигов Пауәрса посредством обращения времени и сужения возникшей обратной динамики на K-подалгебру. Наоборот, для әквивалентнъх полупотоков сдвигов минимальная марковская дилатация определяет ДС, которые после обращения времени оказываются эквивалентными К-системами.

Такого рода соответствие выступает как аналог между непрерывными односторонними и двусторонними сдвигами в гильбертовом пространстве.

Автор признателен Б. М. Гуревичу и А. С. Холево за внимание к работе.

\section{СПИСОК ЛИТЕРАТУРЫ}

[1] Emch G. G. // Commun. Math. Phys. 1976. V. 49. P. 191-215. [2] Alicki R., Narnhofer H. // Lett. Math. Phys. 1995. V. 20. P. 231-250. [3] Narnhofer H., Thirring W. // Lett. Math. Phys. 1990. V. 20. P. 231-250. [4] Булинский А. В. // Функц. анализ и его прилож. 1995. T. 29. C. 64-67. [5] Powers R. T. // Canad. J. Math. 1988. V. 40. P. 86-114. [6] Kummerer B. // J. Funct. Anal. 1985. V. 63. P. 139-177. [7] Kummerer B., Shroeder W. // Commun. Math. Phys. 1983. V. 90. P. 251-262. [8] Accardi L., Cecchini C. // J. Funct. Anal. 1982. V. 45. P. 245-273. [9] Arveson W., Kishimoto A. // Proc. Amer. Math. Soc. 1992. V. 116. P. 769-774. [10] Булинский А. В. // Междунар. конф. "Функциональные пространства, теория приближений, нелинейный анализ" (посвящ. 90-летию акад. С. М. Никольского). Тезисы докл. М.: Изд-во ПАИМС, 1995. С. 67-68. 